\title{
Impact of flux gap upon dynamic resistance of a rotating HTS flux pump
}

\author{
Zhenan Jiang ${ }^{1}$, Chris W B umby ${ }^{1}$, Rodney A Badcock ${ }^{1}$, Hae-Jin Sung ${ }^{2}$, Nicholas J \\ Long $^{1}$, Naoyuki Amemiya ${ }^{3}$ \\ ${ }^{1}$ Robinson Research Institute, Victoria University of Wellington, 69 Gracefield Road, PO \\ Box 33-436, Lower Hutt 5046, New Zealand \\ ${ }^{2}$ Changwon National University, 20 Changwondaehak-ro, Uichang-gu, Changwon-si, \\ Gyeongsangnam-do, Republic of Korea \\ ${ }^{3}$ Graduate School of Engineering, Kyoto University, Kyoto-Daigaku-Katsura, Nishikyo, \\ Kyoto 615-8510, Japan
}

E-mail:zhenan.jiang@vuw.ac.nz

\begin{abstract}
:
HTS flux pumps enable superconducting currents to be directly injected into a magnet coil without the requirement for thermally-inefficient current leads. Here, we present results from an experimental mechanically-rotating HTS flux pump employing a coated-conductorstatorand operated at $77 \mathrm{~K}$. We show the effect of varying the size of the flux gap between the rotor magnets and coated conductor stator from $1 \mathrm{~mm}$ to $7.5 \mathrm{~mm}$. This leads to a corresponding change in the peak applied perpendicular magnetic field at the stator from approximately $350 \mathrm{mT}$ to $50 \mathrm{mT}$. We observe that our experimental device ceases to maintain a measurable output at flux gaps above $7.5 \mathrm{~mm}$, which we attribute to the presence of screening currents in the stator wire.

We show that our mechanically-rotating flux pump is well described by a simple circuit model which enables the output performance to be described using two simple parameters, the open-circuit voltage $V_{\mathrm{oc}}$ and the internal resistance, $R_{\mathrm{d}}$. Both of these parameters are found to be directly proportional to magnet-crossing frequency and decrease with increasing flux gap. We show that the trend in $R_{\mathrm{d}}$ can be understood by considering the dynamic resistance experienced at the stator due to the oscillating amplitude of the applied rotor field. We adopt a literature model for the dynamic resistance within our coated-conductor stator and show that this gives good agreement with the experimentally measured internal resistance of our flux pump.
\end{abstract}


Keywords: HTS Flux pump, Dynamic resistance, coated conductors, superconducting dynamo PACS: 84.71.-b, 84.71.Mn

\section{Introduction}

Flux pump devices enable large electrical currents to be injected into a superconducting circuit without the requirement for normal-conducting current leads which bridge between the cryogenic environment and room temperature. The demonstration of an efficient HTS flux pump could therefore substantially reduce the system heat load incurred whilst energising high- $T_{\mathrm{c}}$ superconducting coils and magnets [1-5].

Previous reports have successfully demonstrated mechanically-rotating flux pumps which employ a coated-conductor HTS stator, and these devices have been used to inject large currents into HTS magnet coils [6-10]. Recently [10], we have shown that we can describe the operation of such devices using a simple electrical circuit model (Figure 1), in which the flux pump is considered to be a voltage source with an effective internal resistance, $R_{\mathrm{d}}$. This internal resistance is due to Dynamic resistance, a well-known phenomenon [11-16] which occurs in a type-II superconductor carrying a DC transport current whilst simultaneously experiencing an alternating imposed magnetic field. The other key parameter to describe the operation of these devices is the open-circuit DC voltage, $V_{\mathrm{oc}}$, which is generated across the flux pump. This is a time-averaged value, as the inductance of the series-connected coil acts to integrate the emf pulse series developed as each rotor magnet passes the coated-conductor stator. (This occurs because the circuit in Figure 1 has a time-constant that is much larger than the pulse frequency). Together, $R_{\mathrm{d}}$ and $V_{\text {oc }}$, enable a full description of the electrical output of this class of flux pumps. During operation, a superconducting coil is connected to the flux pump via low resistance soldered contacts $\left(R_{\mathrm{c}}\right.$ within figure 1$)$. The superconducting coil can therefore be regarded as a separate circuit element obeying its own constitutive equation. This is conceptually different from the situation where a topologically-continuous superconducting bulk or film is magnetized by a time-dependent field $[17,18]$, as the latter case does not require a continuous DC voltage to maintain the circulating current.

In future applications of a mechanically-rotating flux pump, a preferred scenario would allow the rotor to be located at room temperature (i.e. outside the cryostat), whilst the coated-conductor stator is connected to an HTS coil that is located within the cryogenic environment. In such cases, flux pumping would need to take place across a cryostat wall placed in the flux gap between the rotor and the coated-conductor stator. It is therefore important to understand how the operating output of the flux pump will vary as the flux gap of the device is changed. There have been no reports dealing with this issue. Here, we report on the operational performance of our mechanically-rotating flux pump as the flux gap between the rotor magnets and coatedconductor stator is varied between $1 \mathrm{~mm}$ and $7.5 \mathrm{~mm}$. These gap values correspond to a change in the peak applied perpendicular magnetic field from well above the effective AC penetration fields $[19,20]$ of the coated-conductor stator, down to magnetic fields which are of a similar magnitude. We describe the observed effect on the flux pump output in terms of the dependence of the open circuit voltage and dynamic resistance upon the size of the flux gap. 
Figure 1 shows a simple equivalent circuit model for our HTS flux pump which includes the internal resistance of the HTS stator due to dynamic resistance. The superconducting coil is modelled as a pure inductance. The governing equation for the flux pump circuit can be written [10] as,

$$
L \frac{d I}{d t}+I R=V_{\mathrm{oc}}
$$

where the total resistance is given by $R=R_{\mathrm{c}}+R_{\mathrm{d}}$, with $R_{\mathrm{c}}$ denoting the contact resistance of soldered joints within the HTS circuit, and $R_{\mathrm{d}}$ denoting the dynamic resistance experienced at the flux pump stator. $L$ is the coil inductance, $V_{\text {oc }}$ is the open-circuit voltage. We can solve for the current, $I$, as a function of time, $t$, to obtain

$$
I(t)=\frac{V_{\mathrm{oc}}}{R}\left(1-e^{-\frac{R}{L} t}\right)=I_{\mathrm{sc}}\left(1-e^{-\frac{R}{L} t}\right)
$$

and the voltage across the coil is given as

$$
V_{\text {coil }}(t)=L \frac{d I}{d t}=V_{\mathrm{oc}} \cdot e^{-\frac{R}{L} t}
$$

where $I_{\mathrm{sc}}=V_{\mathrm{od}} R$ is the short-circuit current, which is the maximum current that can possibly be delivered by the flux pump. The time constant of the circuit shown in Figure $1, \tau$, is equal to $L / R$. For our system, $R_{\mathrm{c}} \approx 1$ $\mu \Omega$, which is much less than $R_{d}$ of our device under the operating conditions studied in this work. Hence we can take $R \approx R_{\mathrm{d}}$, and we see that the contact resistance of soldered joints within our circuit plays no role in determining the limiting current delivered by the HTS flux pump. Instead, the available output voltage from the flux pump is dropped entirely across the coil such that $V \cong V_{\text {coil }}$. Note that if the available output current of the flux pump exceeds the critical current of the superconductor coil, then the approximation of the coil as a pure inductance no longer holds, and equation (3) is not valid - as the coil voltage must follow the $I-V$ relationship of the superconductor coil.

In designing an HTS flux pump to energise a given coil, we therefore need to be able to calculate estimates of $V_{\text {oc }}$ and $R_{\mathrm{d}}$. The output voltage of a superconducting flux pump is often expressed as $V=f \Delta \phi$, where $\Delta \phi$ is the total voltage-generating flux passing through the HTS stator wire per magnetcrossing cycle, and $f$ is the frequency of magnet-crossing [1]. In our previous work, we have shown that the equation of Oomen et al [13], can be adopted to describe the dynamic resistance within the HTS flux pump stator, and this is given in equation (4).

$$
R_{\mathrm{d}}=\frac{4 a l_{\mathrm{eff}} f}{I_{\mathrm{c} 0}}\left(B_{\mathrm{a}}+\frac{B_{\mathrm{a}}{ }^{2}}{B_{0}}\right) \quad \text { for } B_{\mathrm{a}}>>2 B_{\mathrm{th}}
$$


where $2 a$ is the width of the conductor and $l_{\text {eff }}$ is the effective interaction length of the AC magnetic field with the conductor. $I_{\mathrm{c} 0}$ and $B_{0}$ are parameters from the Kim model [21] for critical current,

$I_{\mathrm{c}}=I_{\mathrm{c} 0} /\left(1+B_{\mathrm{a}} / B_{0}\right)$ and $B_{\mathrm{a}}$ is the amplitude of the time-varying magnetic field. This equation is applicable when the amplitude of magnetic field is larger than twice the threshold magnetic field, $B_{\mathrm{th}}$ [13]. $B_{\mathrm{th}}$ describes the minimum applied field at which the sum of transport and screening currents satisfy the condition $|J|=J_{\mathrm{c}}$ at all points within the superconductor. The maximum value of $B_{\mathrm{th}}$ occurs when the DC transport current is zero, at which point it is equal to the penetration field, $B_{\mathrm{p}}$. In this paper we show that this approach provides reasonable approximations for the dynamic resistance of our flux pump across the entire range of operating flux gaps and frequencies. Both $V_{\mathrm{oc}}$ and the effective internal resistance $R_{\mathrm{d}}$, are expected to be proportional to frequency, and to decrease as the applied peak magnetic field at the conductor declines. As the flux gap is increased, the relative changes in these two factors determines the current flowing through the coil (through equation 2).

In this paper we present experimental measurements of $R_{\mathrm{d}}$ and $V_{\text {oc }}$ from our prototype mechanically-rotating flux pump. The physics underlying dynamic resistance is well described [11-16] by conventional AC loss theory. However, at present, the physics underlying the origin of the DC open-circuit voltage in these devices is not so well understood, and there is no equivalent literature.

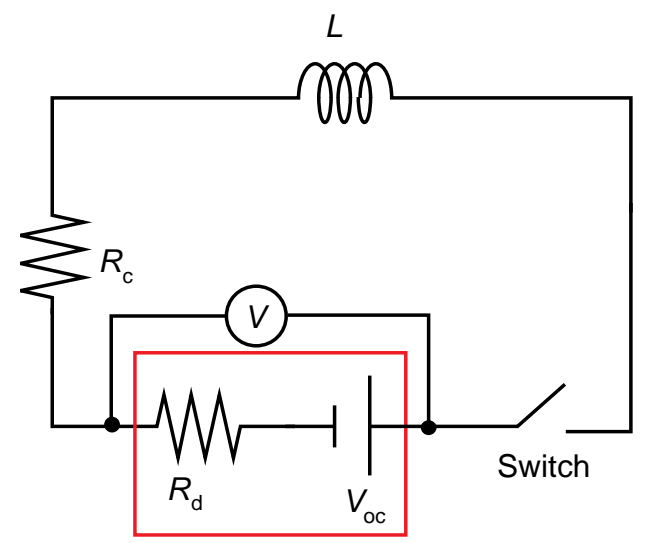

Flux pump

Figure 1. Circuit diagram of a superconducting circuit consisted of a HTS coil and a HTS flux pump 


\section{Experimental set-up}

The experimental set-up of the mechanically rotating coated-conductor flux pump built and studied in this work is shown in Figure 2. In this experimental device, the rotor is composed of two wheels of permanent magnets moving across a coated conductor. Each wheel has an outer diameter of $70 \mathrm{~mm}$, and twelve $\mathrm{NdFeB}$ N38 magnets are equally distributed around the circumference. Each magnet has a diameter of $10 \mathrm{~mm}$ and a height of $10 \mathrm{~mm}$. The coated-conductor stator is $12 \mathrm{~mm}$-wide stabilizer-free SuperPower wire (SF12100) with a self-field $I_{\mathrm{c}}$ of $313 \mathrm{~A}$. The flux gap between the surface of the magnets and superconducting layer, $g$ shown in figure 2(b), varies between $1.0-7.5 \mathrm{~mm}$, which was adjusted in situ using a simple worm drive. During device operation, the rotor and all superconducting components were held at $77 \mathrm{~K}$ through immersion in a liquid nitrogen bath.

The coated-conductor stator is connected to a double pancake coil wound with AMSC coated conductor via soldered joints to form a closed circuit $[6,10]$. The length of the $4 \mathrm{~mm}$-wide AMSC conductor is $40 \mathrm{~m}$, and its self-field $I_{\mathrm{c}}$ is $88 \mathrm{~A}$. The inner and outer diameters of the coil are $50 \mathrm{~mm}$ and $90 \mathrm{~mm}$, respectively, and the total number of turns is 163 . Its inductance, $L$, is measured to be $2.4 \mathrm{mH}$ at $77 \mathrm{~K}$. The measured coil $I_{\mathrm{c}}$ (determined at $1 \mu \mathrm{V} / \mathrm{cm}$ criterion) is measured to be $55 \mathrm{~A}$. The coil current is calculated from calibrated measurements of the axial magnetic field in the centre of the coil, which was measured using a fixed cryogenic hall sensor (Arepoc HHP-NA). The contact resistance in the circuit was obtained from the decay time constant of the coil after the flux pump was de-energised. The total contact resistance between the coil and the coated conductor was found to be $R_{\mathrm{c}}=0.88 \mu \Omega$.

We have found that it is necessary for the $I_{\mathrm{c}}$ of the flux pump stator to be substantially larger than the $I_{\mathrm{c}}$ of the series-connected coil, in order to achieve operating currents which are a significant fraction of the coil $I_{\mathrm{c}}$. This is due to the limiting effect of the internal resistance of the flux pump, which can be seen from equation 4 where $R_{\mathrm{d}}$ becomes large if $I_{\mathrm{c} 0}$ is small.

An Omron R7M-A20030-S1 servo motor is used to power the rotor wheels, which are connected via a thermally-insulating rotating shaft. The rotation speed is adjustable in the range of $0-3000 \mathrm{rpm}$. We used this to obtain experimental operating frequencies from $48 \mathrm{~Hz}$ to $600 \mathrm{~Hz}$. Frequency is defined as the passing frequency of the rotor magnets across the coated conductor stator, i.e. $240 \mathrm{rpm}$ of the motor is equivalent to $48 \mathrm{~Hz}(240 / 60 \times 12)$, because each wheel has 12 magnets. Two voltage taps are attached at the centre of the surface of the coated conductor (the surface of the substrate side). The distance between the voltage taps is $50 \mathrm{~mm}$ (see figure 2(b)). Another two voltage taps also are attached on the ends of the coated-conductor coil. There was little difference in the measured voltage values using the two sets of the voltage taps. In this work, the voltage taps attached on the conductor surface are used. Voltage measurements were acquired using a sample integration time of $0.2 \mathrm{~s}$, which is substantially longer than the cycle period of the rotor magnets at all frequencies studied. 


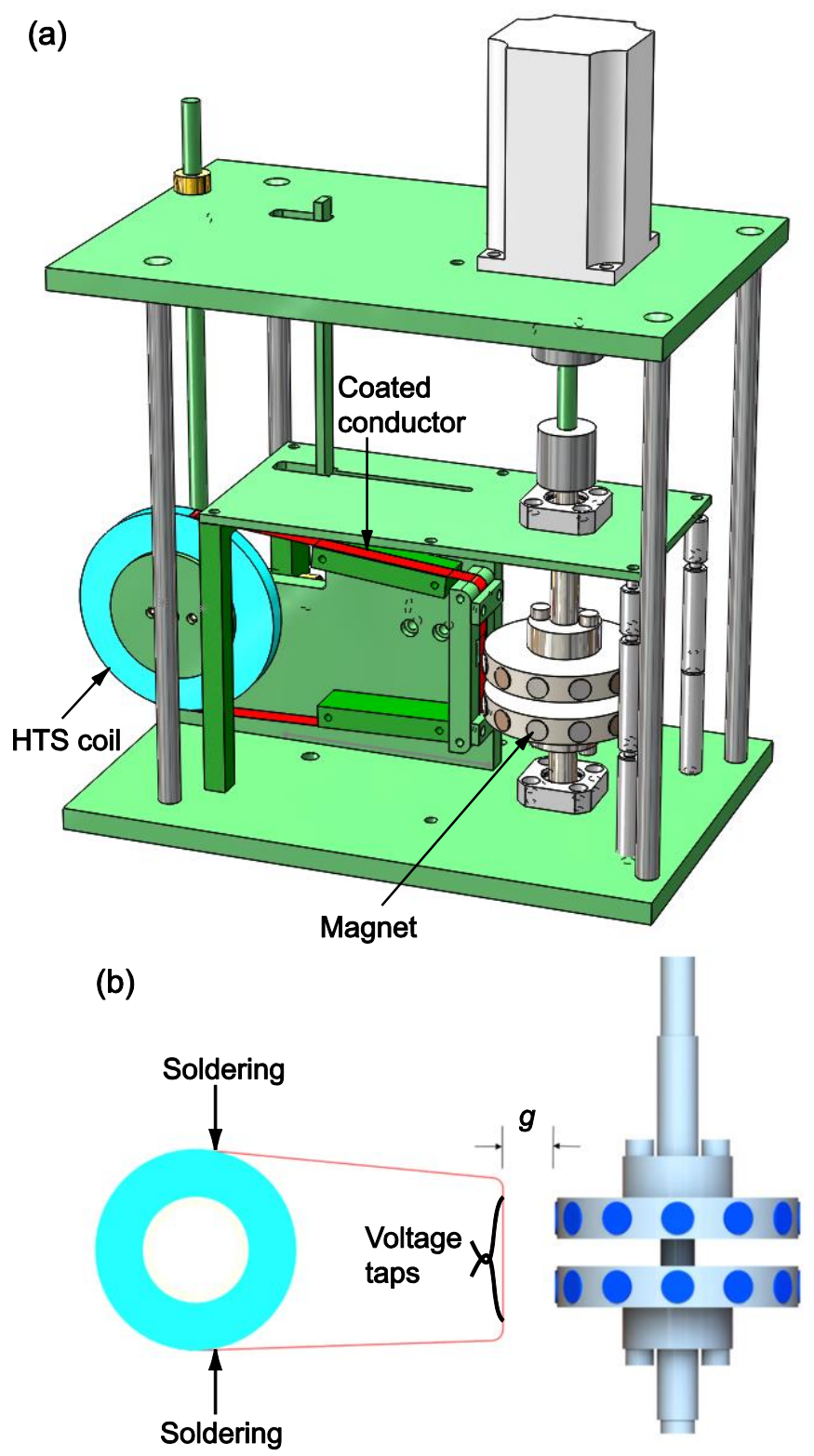

Figure 2. (a) Experimental set-up of the flux pump, (b) side view of the HTS coil, coated conductor stator, and rotor consisting of two wheels of magnets 


\section{Experimental results}

Figure 3 shows a comparison of the injected coil currents obtained when operating the flux pump at $600 \mathrm{~Hz}$ using various flux gaps. The current obtained at each flux gap is plotted as a function of time. We observe that for $t \gg \tau$ the coil current saturates at a value, $I_{\mathrm{sat}}$, which is determined by resistive losses in the circuit due to the $I-V$ relationship of the coil. $I_{\text {sat }}$ is similar to the critical current of the coil at small flux gaps, and decreases with increasing flux gap. At the largest gap, $g=7.5 \mathrm{~mm}$, a coil current of $7.5 \mathrm{~A}$ had been injected after 1 hour, at which point the experiment was terminated. We did not observe measurable current injection at flux gaps larger than $7.5 \mathrm{~mm}$.

Figure 4 shows the corresponding output voltages measured across the flux pump during the same series of experiments. The output voltage at each flux gap exhibits the expected exponential decay described by equation (3), with the added factor that at long times and smaller gaps (1-3 $\mathrm{mm})$, the voltages saturate at a value which is again defined by the $I-V$ curve of the coil. The time constant, $\tau=L / R$, can be obtained from the initial gradient of each line in figure 4 and we see that $\tau \gg>1 / f$ in all cases. We observe that $\tau$ increases as the flux gap increases, which is a result of the reduction in $R_{\mathrm{d}}$ as the peak applied field at the stator decreases. Similarly, the voltage at zero time $V(t=0)=V_{\text {oc }}$, decreases with increasing flux gap, again reflecting the reduced applied flux which passes through the stator as the distance between the stator and rotor magnets is increased. $V_{\text {oc }}$ changes very rapidly with distance at smaller gaps, and the absolute value is susceptible to small errors in length measurement in this regime. 


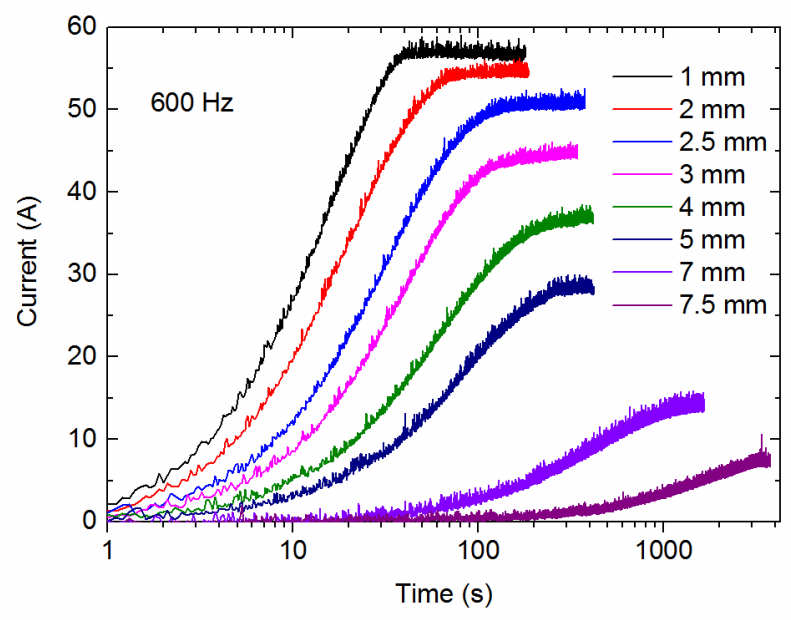

Figure 3. Comparison of coil currents at $600 \mathrm{~Hz}$ for various flux gaps

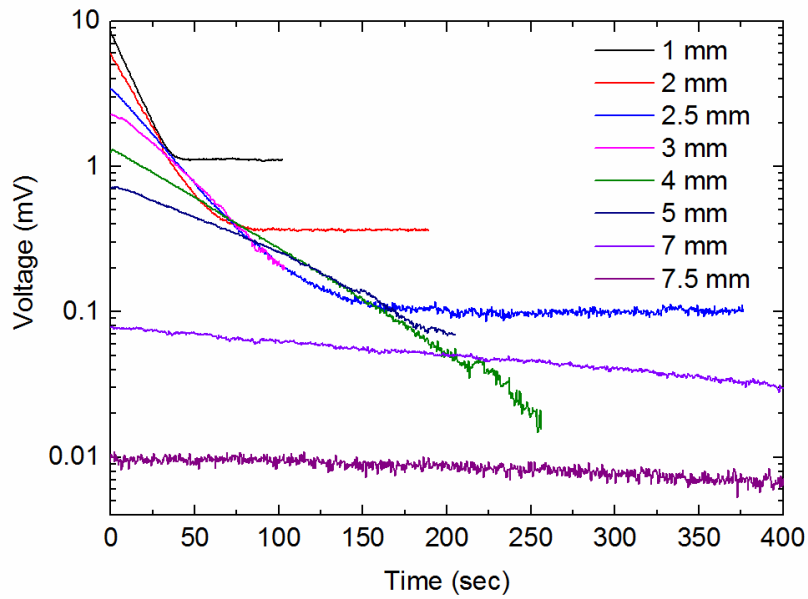

Figure 4. Comparison of output voltages from the flux pump (which are equal to voltages across the coil) at $600 \mathrm{~Hz}$ for various flux gaps 


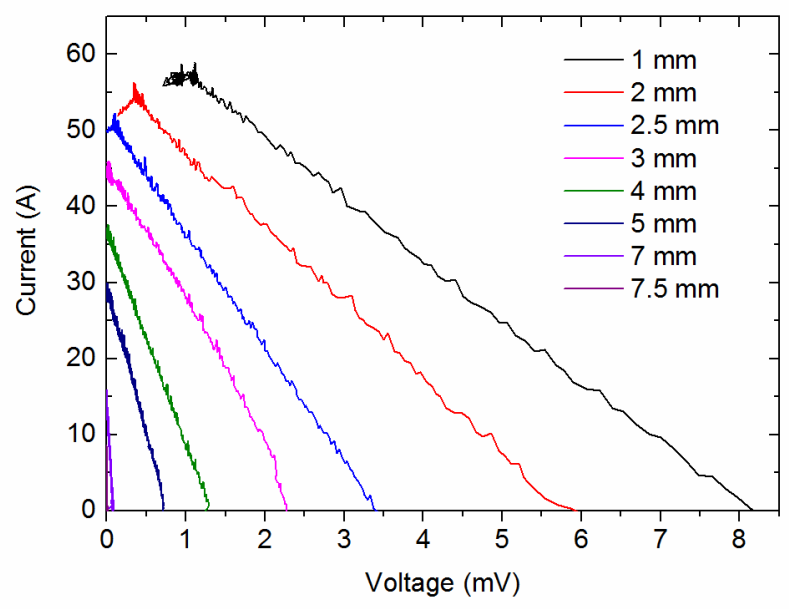

Figure 5. Comparison of $V-I$ relationships at $600 \mathrm{~Hz}$ for various flux gaps

Figure 5 shows the same experimental data as given in figures 3 and 4, but now plotted in terms of the output current, $I$, versus the output voltage, $V$, of the flux pump operating at each flux gap. A straight line relationship between output current and voltage is observed, which clearly demonstrates that the internal resistance of the flux pump stator is independent of current in each case (as $R_{\mathrm{d}}=-\mathrm{d} V / \mathrm{d} I$ following the sign convention used in this plot). Experimental values for the open-circuit voltage, $V_{\text {oc }}$ and short-circuit current, $I_{\mathrm{sc}}$ can also be extracted from this plot as these values are given by the intercepts of each line on the $V$ and $I$ axes respectively. Similar plots can be produced for each operating frequency, and in this manner we have obtained experimental values for $V_{\mathrm{oc}}, R_{\mathrm{d}}$ and $I_{\mathrm{sc}}$ at operating frequencies between $48 \mathrm{~Hz}$ and $600 \mathrm{~Hz}$ at each flux gap studied. These values are shown in figure 6 , where they are linearly normalised against operating frequency.

At each flux gap, we see that the frequency-normalised values closely agree across all operating frequencies measured. This confirms that $V_{\text {oc }}$ and $R_{\mathrm{d}}$ are linearly proportional to frequency across the entire operating range of our device, as predicted from equation 4 and observed in our previous work [10]. The observed values of $V_{\text {oc }}$ and $R_{\mathrm{d}}$ both decrease as the flux gap increases, which is a direct result of the decrease in the applied magnetic field as the rotor magnet moves further away from the HTS stator. Results obtained at $600 \mathrm{~Hz}$ show that $V_{\mathrm{od}} / f$ approaches zero for $g \geq 7.5 \mathrm{~mm}$, and as a result no current can be pumped at flux gaps larger than this value, regardless of whether higher operating frequencies could be employed. Figure 6 (c) also shows that the maximum output current of the flux pump, $I_{\mathrm{sc}}$, decreases with increasing flux gap across the full range of gap distances studied, which reflects the fact that $V_{\text {oc }}$ decreases faster than $R_{\mathrm{d}}$ as the gap increases. 


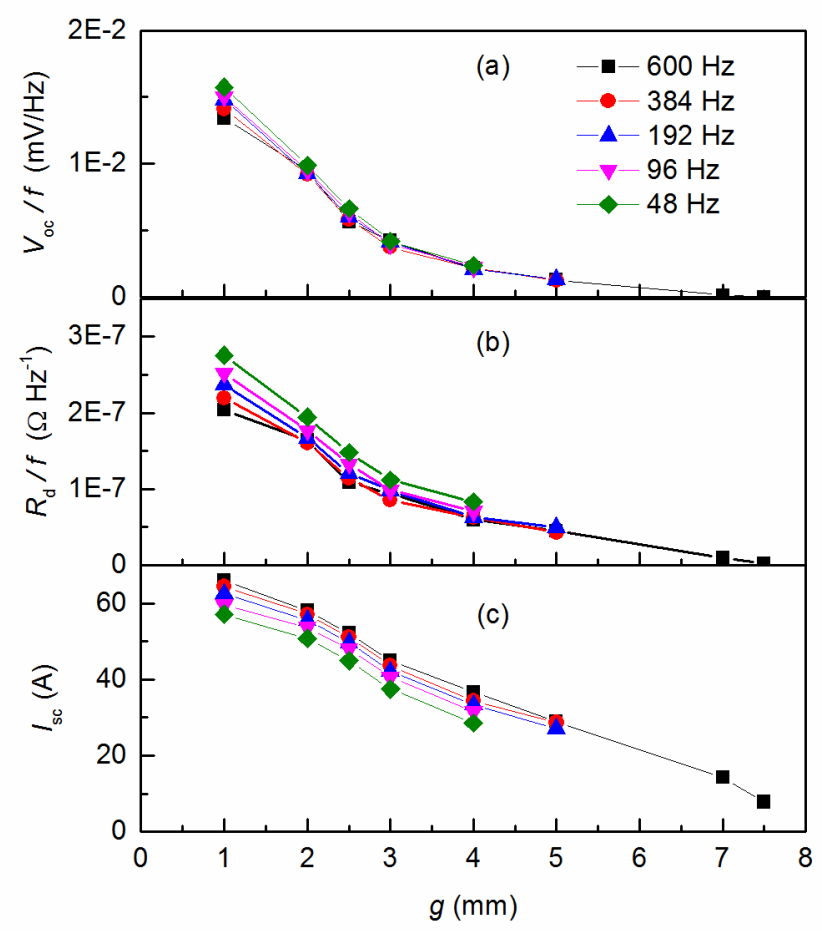

Figure 6. Experimental values plotted against flux gap, $g$, for: (a) open-circuit voltage, $V_{\text {oc }}$ (normalised by magnet-crossing frequency, $f$ ); (b) internal resistance of the flux pump stator, $R_{\mathrm{d}}$, (normalised by magnetcrossing frequency, $f$ ); and (c) short-circuit current, $I_{\mathrm{sc}}$.

\section{Estimation of fields and modelling dynamic resistance}

To gain further insight into the changes in $V_{\mathrm{oc}}, R_{\mathrm{d}}$ and $I_{\mathrm{sc}}$ we need to understand the amplitude and profile of the applied magnetic fields experienced at the coated-conductor stator during the operating cycle of the flux pump. The relative size and spacing of the permanent magnets used in our flux pump design mean that, different parts of the conductor experience different magnetic fields as the rotor magnets move across the coated conductor stator. Figure 7 shows the calculated applied perpendicular magnetic field profiles across the coated conductor for different $g$ values along the centre-line of the magnet in two conditions: (i) when a rotor magnet is directly aligned with the centre of the HTS stator (see figure 7(a)); and (ii) when the centre of the gap between two adjacent rotor magnets is directly aligned with the centre of the HTS stator (see figure 7(b)). These profiles were obtained by finite-element calculation using Opera [22] software. Figure 7(a) 10 
shows the maximum applied perpendicular magnetic field experienced at the stator, and figure 7(b) shows the minimum applied perpendicular magnetic field.
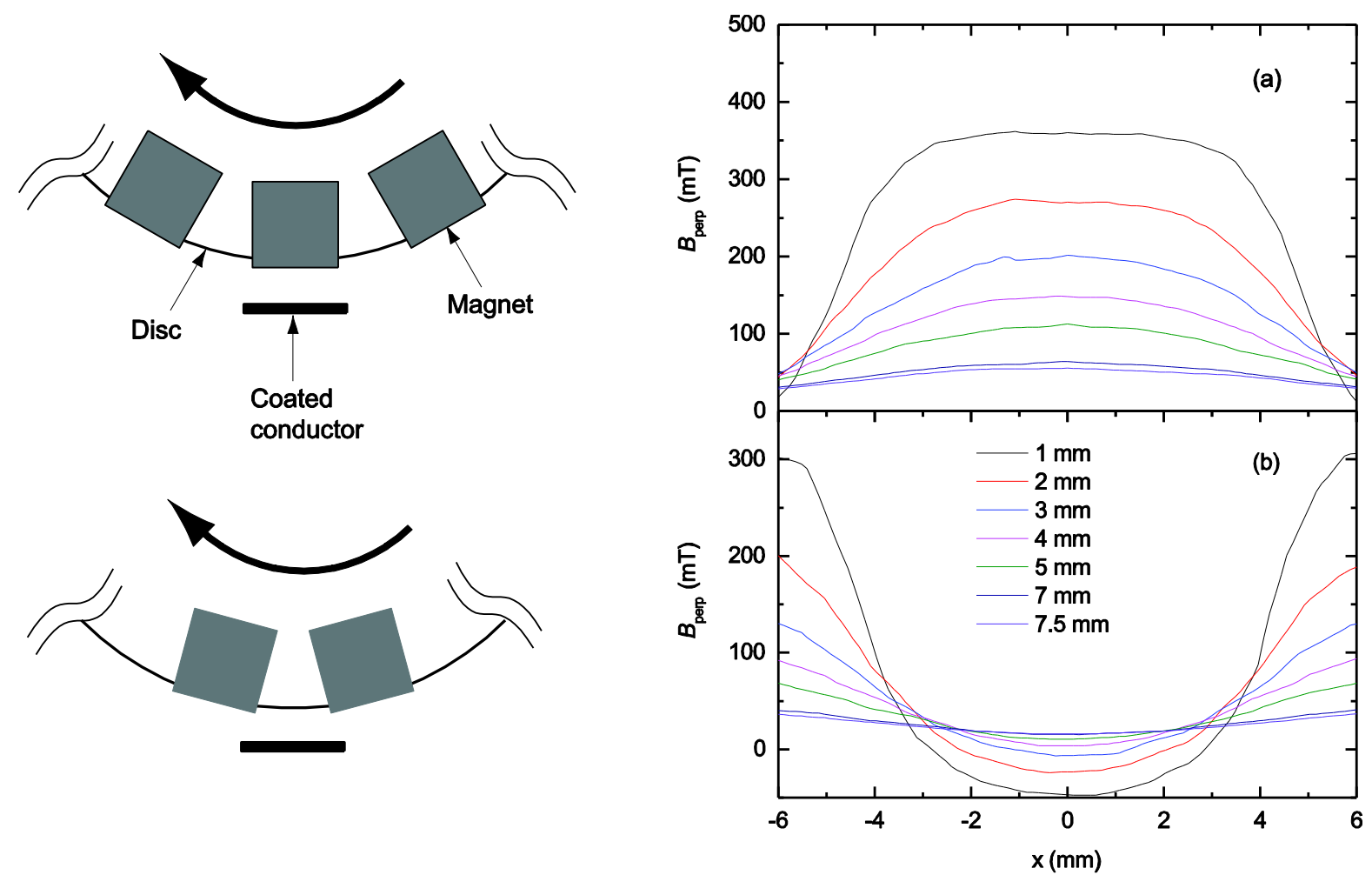

Figure 7. Magnetic field profiles across the coated conductor, (a) the magnets are on top of the conductor, (b) the magnets move away from the conductor

Using these calculated values, we are now able to consider the trend in open circuit voltage and short circuit current as a function of the peak applied perpendicular magnetic field at the stator (e.g. $B_{\text {perp }}(\mathrm{x}=0)$ from figure 7(a)). Figure 8(a) shows that the frequency-normalised open circuit voltage increases approximately linearly with peak applied field for fields above a minimum threshold field of $\approx 50 \mathrm{mT}$. The flux pump is not observed to operate when the applied peak field drops below this threshold value, and we interpret this value to represent the effective penetration field at which flux is able to fully pass through the coated-conductor stator. A simplified schematic of this situation is shown in figure 9, which shows the perpendicular magnetic field profile across an HTS conductor as the applied field, $B_{\text {perp }}$, is cycled between 0 and $>2 B_{\mathrm{p}}$. When $B_{\text {perp }}=0$ the HTS wire retains a remnant field with a maximum value of $B_{\mathrm{p}}$ at its centre line. The field at the centre of the wire remains constant until the applied field exceeds $2 B_{\mathrm{p}}$. Hence for applied 
fields less than $2 B_{\mathrm{p}}$, flux-lines at the centre of the stator strip are frozen, and flux cannot traverse the wire. This implies that for a cycle with an amplitude of $\left\langle 2 B_{\mathrm{p}}\right.$, any flux which is expelled from the superconductor must exit the wire across the same boundary as it entered. In this case, it follows that the time-averaged output voltage across the stator therefore equates to zero. However, once the applied field exceeds $2 B_{\mathrm{p}}$, then flux is able to flow across the centre-line of the coated-conductor. Dipole interactions with the travelling rotor magnets leads to flux being "dragged" through the coated-conductor wire, entering at one edge and exiting at the opposite edge. We surmise that it is this action which gives rise to the observed DC output voltage from our device. The penetration field, $B_{\mathrm{p}}$ can be determined from the maxima of the magnetic ac loss for an HTS wire [20]. For the coated-conductor HTS wire used in this experiment, this ac loss is known [23-26] to closely follow the strip expression of Brandt and Indenbom [19], and is shown in figure A1 (Appendix). We find that the ac loss maxima occurs at $\sim 25 \mathrm{mT}$, indicating that $2 B_{\mathrm{p}} \sim 50 \mathrm{mT}$, which is consistent with our observations.

The schematic shown in figure 9 considers a homogeneous field applied to a superconducting slab. This is a substantial simplification of the situation experienced within our flux pump, where the rotor magnets actually impose an inhomogeneous applied field gradient across the stator strip. Nonetheless, the requirement remains that in order for flux to traverse the coated-conductor wire, the peak applied field must be more than twice the maximum remnant field present at the centre of the wire [27]. Once this condition is satisfied, a time-averaged DC output voltage is developed by the flux pump, and this voltage drives a transport current through the series-connected coil according to equations 1 and 2.

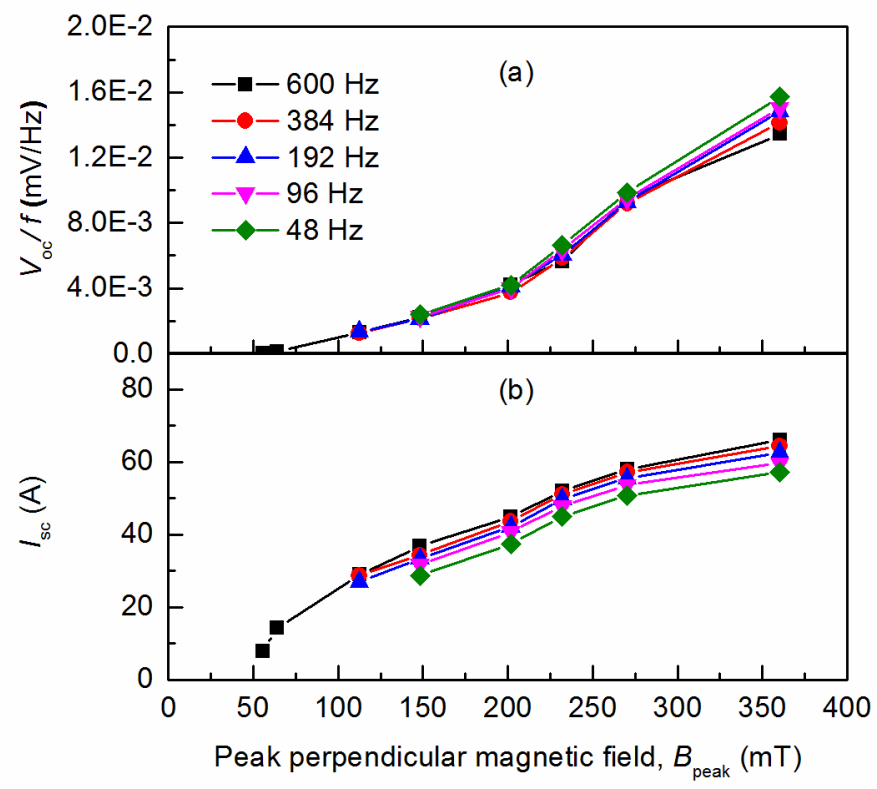


Figure 8. Plots versus the peak perpendicular magnetic field applied at the stator showing (a) open-circuit voltage normalised to operating frequency, $V_{\mathrm{oc}} f f$; and (b) short-circuit current, $I_{\mathrm{sc}}$.

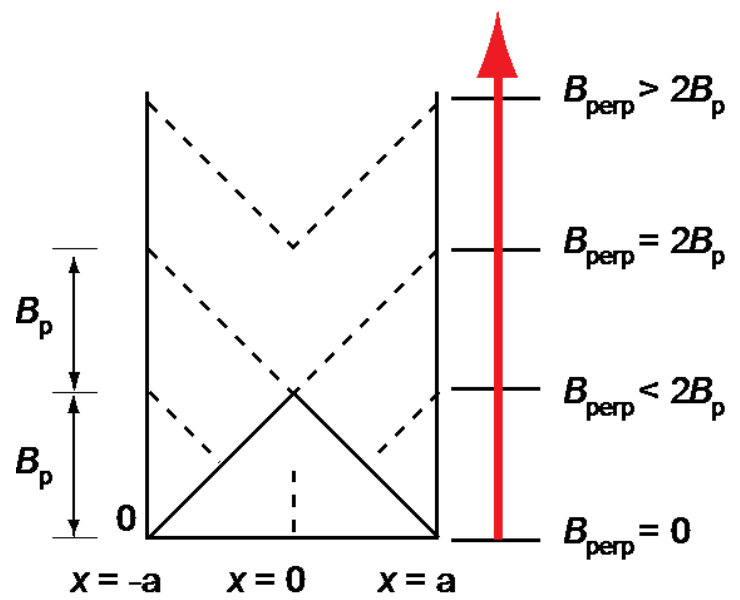

Figure 9. Schematic illustration of the perpendicular magnetic field profile across an HTS conductor as the applied perpendicular field, $B_{\text {perp }}$ is cycled from zero to $>2 B_{\mathrm{p}}$. Note that for $B_{\text {perp }} \leq 2 B_{\mathrm{p}}$, the field at $x=0$ is unchanged.

Figure 8(b) shows that the short-circuit current also approaches zero when $B_{\text {peak }} \approx 50 \mathrm{mT}$, which is unsurprising as the impedance of the circuit can only be overcome if there is an output voltage present across the flux pump. We see that the short-circuit current drops steadily as the flux gap increases, indicating that $R_{d}$ drops more slowly than $V_{\text {oc }}$ with decreasing $B_{\text {peak. }}$. This is consistent with equation 4 and the trend shown in figure 8(a) as $R_{\mathrm{d}}>0$ at $B_{\text {peak }}=2 B_{\mathrm{p}}$, and $R_{\mathrm{d}}$ retains a non-zero positive value at all amplitudes of applied field which are greater than zero. We emphasise that the oscillation amplitude of the applied field, $B_{\mathrm{a}}$, which is used in equation 4 is not the same as the absolute magnitude of the field. We can define the oscillation amplitude of the applied magnetic field, $B_{\mathrm{a}}$ in terms of the maximum and minimum fields averaged across the full width of the stator. This is expressed in the following manner: $B_{\mathrm{a}}=\Delta B / 2=\left(\bar{B}_{\text {max }}-\bar{B}_{\text {min }}\right) / 2$ where $\bar{B}$ is calculated from figure 7(a) or (b) respectively through

$$
\bar{B}=\frac{1}{2 a} \int_{-a}^{a} B(x) \mathrm{d} x
$$

where $2 a$ is the width of the stator, such that $a=6 \mathrm{~mm}$ in the case considered here.

Using this definition of $B_{\mathrm{a}}$, we can now plot our experimentally measured values for $R_{\mathrm{d}} / f$ against $B_{\mathrm{a}}$, as shown in figure 10. In this figure we also show calculated values for $R_{\mathrm{d}}$ given by equation (4), where we 
have used values of $I_{\mathrm{c} 0}=313 \mathrm{~A}$, and $B_{0}=75 \mathrm{mT}$, which have been obtained from independent measurements of the stator wire (Appendix: Figure A2). We take the effective magnetic length, $l_{\mathrm{eff}}$, to be twice the average diameter of rotor magnet (as our device includes two rotor wheels), and we obtain this from the area average over the full width of the stator wire such that $l_{\mathrm{eff}} / 2=\pi r^{2} / 2 a$.

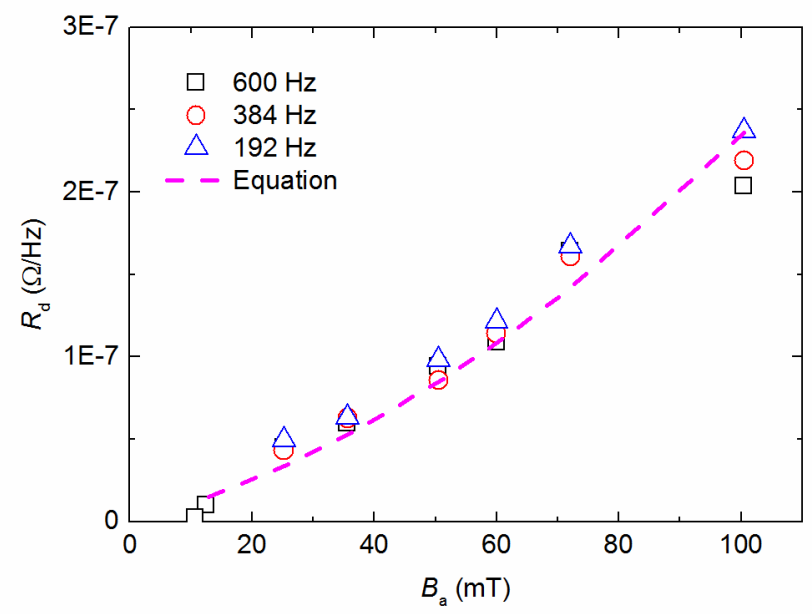

Figure 10. Experimental $R_{\mathrm{d}}$ values plotted against the average oscillation amplitude of the applied perpendicular magnetic field, $B_{\mathrm{a}}$. Also shown are calculated values determined from equation (4).

For values which correspond to gaps $<7 \mathrm{~mm}$, we see very good agreement between the calculated and experimentally measured values for $R_{\mathrm{d}}$. As observed in figure 8 , at flux gaps of $>7 \mathrm{~mm}$ the flux pump is operating at the limit of its capabilities, such that $B_{\text {peak }} \approx 50 \mathrm{mT} \approx 2 B_{\mathrm{p}}$ which is equal to the value of $2 B_{\text {th }}$ when the transport current is zero [13]. As such, the assumed conditions for equation (4) cease to be valid and as a result our model overestimates the value of $R_{\mathrm{d}}$ in this regime. However, the efficacy of flux pumping in this very low-field regime is extremely weak, and practical future devices will be required to operate in the higher-field regime. At these higher applied magnetic field amplitudes our model shows good agreement with experiment, and hence has practical utility for the design and optimisation of future coated-conductor flux pumps.

\section{Conclusion}

In this work we have described an experimental mechanically-rotating HTS flux pump and demonstrated its use to energise an HTS coil, through the injection of current of up to $58 \mathrm{~A}$. We have shown that this flux 
pump can be described by a simple circuit model in which the internal resistance and open-circuit voltage are directly proportional to frequency across the entire operating range. We have also shown that the flux pump output exhibits a strong dependence upon the flux gap between the rotor magnets and the coated-conductor stator which is due to the reduction in $V_{\text {oc }}$ as the flux gap increases. This reduction is a result of the changes in the magnitude and profile of the applied magnetic field experienced at the stator. The flux pump presented here is capable of injecting current into our test coil at flux gaps of up to $7.5 \mathrm{~mm}$. At flux gaps above $7.5 \mathrm{~mm}$ the device does not develop an output voltage, regardless of the operating frequency. This corresponds to a threshold value for the peak applied perpendicular magnetic field of $\sim 50 \mathrm{mT} \approx 2 B_{\mathrm{p}}$. We attribute this effect to the presence of shielding currents within the stator, such that $B_{\text {peak }}$ must exceed $2 B_{\mathrm{p}}$ in order for flux to traverse the full width of the coated-conductor stator.

Using our experimental results we have then validated our previously proposed model, which describes the observed internal resistance of the pump in terms of the dynamic resistance of the stator in the presence of the oscillating rotor field. We have shown that this model provides good agreement with the experimentally measured values for the internal resistance of the flux pump across the entire operating range of our device. We observe that the maximum possible output current which can be delivered by our flux pump, $I_{\mathrm{sc}}$, drops steadily with increasing flux gap across the entire operating range of our device. This is a result of the fact that the internal resistance, $R_{\mathrm{d}}$ drops more slowly with decreasing flux gap than does $V_{\text {oc }}$. This can be understood in terms of the fact that dynamic resistance is non-zero and positive for all values of an applied oscillating magnet field which are greater than zero, whilst the flux pump only generates a DC voltage when the peak field is greater than the threshold value $2 B_{\mathrm{p}} \approx 50 \mathrm{mT}$.

Our results provide new insights into the operating principle of mechanically-rotating HTS flux pumps, but the underlying physical origin of $V_{\text {oc }}$ in these devices still remains a subject of ongoing study. It is clear that the motion of penetrating flux in the high-field region directly beneath the rotor magnet induces an instantaneous voltage within the HTS stator wire, and that the magnitude of this voltage is proportional to the rate at which this occurs (i. e. the rotor frequency). However, the geometry of the device is topologically identical to an AC generator [28], so the origin of the observed DC voltage cannot be explained by simple application of Faraday's law of induction [29]. Our observations suggest that shielding currents play an important role - particularly at large flux gaps. Detailed finite-element modelling of this effect will be required to confirm this, but such work lies beyond the scope of this paper.

In justifying our simple electrical circuit model we have noted that the presence of normal-conducting soldered joints between the flux pump and coil allow the se components to be considered as separate circuit elements. An interesting question is to consider - what would happen if we had a truly persistent superconducting joint between the flux pump and the coil? In fact, we expect that during flux pump operation the observed current within the circuit (and output voltage across the flux pump) would only alter minimally, as the internal resistance of the flux pump is already much larger than the joint resistances in the circuit studied (i. e. $R_{\mathrm{d}} \gg R_{\mathrm{c}}$ ). However once the flux pump is turned off (such that the rotor is stationary), then current decay within the circuit would be drastically reduced by a superconducting joint and the coil could be operated in a persistent mode.

Finally, we note that a key motivation for this work was to explore the possibility of locating the flux 
pump rotor outside the cryogenic envelope. However, a flux gap of $<7 \mathrm{~mm}$ presents some challenges for the accommodation of a mechanically-sound, thermally-insulating cryostat wall which could enable this to occur. We anticipate new designs of HTS flux pumps which can operate across larger flux gaps by incorporating magnetic circuits to concentrate and focus the applied magnetic field.

\section{Acknowledgments}

This work was supported by the New Zealand MBIE Contract No. RTVU1402.

\section{Appendix}

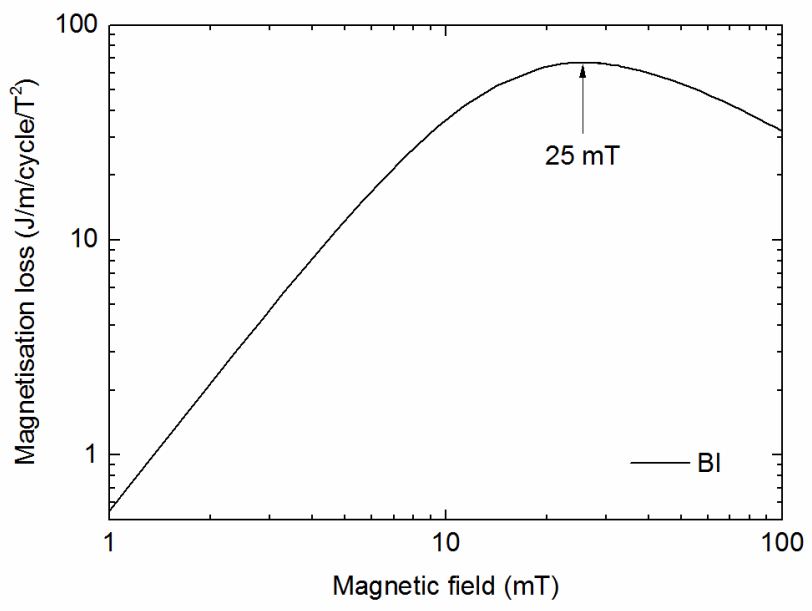

Figure A1. Plot of calculated AC magnetization loss [19], $\Gamma=Q_{\mathrm{Bl}} / B_{\mathrm{a}}{ }^{2}$ (denoted with $\mathrm{BI}$ ), used to define the penetration field, $B_{\mathrm{p}}$ in our coated-conductor HTS Superpower stator wire [20]. The maxima of $\Gamma$ occurs at an applied field equal to $B_{\mathrm{p}}$, which in this case is found to be approximately $25 \mathrm{mT}$. 


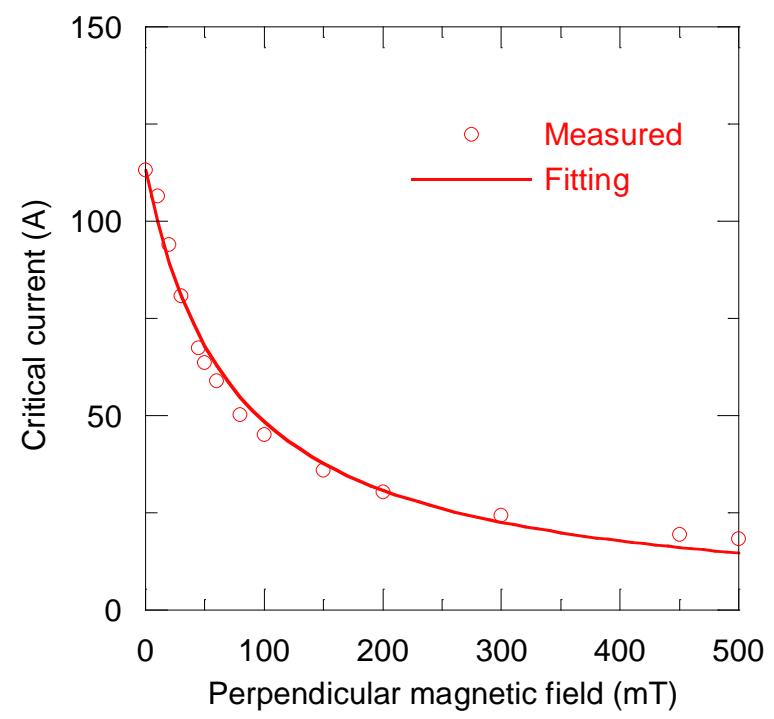

Figure A2. Plot showing the dependence of the critical current at $77 \mathrm{~K}, I_{c}$, upon the applied perpendicular magnetic field for a coated-conductor HTS Superpower wire similar to the stator wire in our Flux pump. $I_{\mathrm{c}}$ was measured at the $1 \mu \mathrm{V} / \mathrm{cm}$ criterion on a $4 \mathrm{~mm}$-wide sample of wire (note that the Flux pump stator wire is $12 \mathrm{~mm}$ wide). The fitting curve shows a fit to the measured data for the Kim equation $I_{\mathrm{c}}=I_{\mathrm{c} 0} /\left(1+B_{\mathrm{a}} / B_{0}\right)$. From this fit we obtain a value of $B_{0}=75 \mathrm{mT}$.

\section{References}

[1] van de Klundert L J M and ten Kate H H J 1981 Cryogenics 21 195-206

[2] van de Klundert L J M and ten Kate H H J 1981 Cryogenics 21 267-77

[3] Oomen M P, Leghissa M, Ries G, Proelss N, Neumueller H W, Steinmeyer F, Vester M and Davies F 2005 IEEE Trans. Appl. Supercond. 15 1465-8

[4] Ishmael S, Goodzeit C, Masson P, Meinke R and Sullivan R 2008 IEEE Trans. Appl. Supercond. 18 693-6

[5] Nakamura T, Sugano M, Doi T and Amemiya N 2010 IEEE Trans. Appl. Supercond. 20 1033-6

[6] Hoffmann C, Pooke D and Caplin A D 2011 IEEE Trans. Appl. Supercond. 211628

[7] Hoffmann C, Walsh R, Karrer-Mueller E and Pooke D 2012 Phys. Proc. 361324

[8] Walsh R M, Slade R, Pooke D and Hoffmann C 2014 IEEE Trans. Appl. Supercond. 244600805

[9] Coombs T A, Fagnard J F and Matsuda K. 2014 IEEE Trans. Appl. Supercond. 248201005

[10] Jiang Z, Hamilton K, Amemiya N, Badcock R and Bumby C W 2014 Appl. Phys. Lett., 105112601 
[11] Andrianov V V, Zenkevitch V B, Kurguzov V V, Sytchev V V, and Ternovskii F F 1970 Sov. Phys. JETP $31815-9$

[12] Ogasawara K, Yasukochi K, Nose S and Sekizawa H 1976 Cryogenics 16 33-8

[13] Oomen M P, Rieger J, Leghissa M, ten Haken B and ten Kate H H J 1999 Supercond. Sci. Technol. 12 $382-7$

[14] Ciszek M, Knoopers H G, Rabbers J J, ten Haken B and ten Kate H H J 2002 Supercond. Sci. Technol. 15 1275-80

[15] Ryu K, Song H J, Kim H J and Seong K C 2006 IEEE Trans. Appl. Supercond. 16 1011-4

[16] Duckworth R C, Zhang Y F, Ha T, and Gouge M J 2011 IEEE Trans. Appl. Supercond. 21 3251-6

[17] Wang W, Zhang M, Huang Z, Zhai Y, Zhong Z, Spaven F, Baghdadi M and Coombs T 2014 IEEE. Trans. Appl. Supercond. 244600304

[18] Wang W, Spaven F, Zhang M, Baghdadi M and Coombs T 2014 Appl. Phys. Lett. 104032602

[19] Brandt E H and Indenbem 1993 Phys. Rev. B 48 12893-906

[20] Iwakuma M, Toyota K, Nigo M, Kiss T, Funaki K, Iijima Y, Saitoh T, Yamada Y and Shiohara Y 2004 Physica C 412-414 983-91

[21] Kim Y B, Hempstead CF and Strnad A R 1962 Phys. Rev. Lett. 9 306-9

[22] http:/operafea.com/

[23] Nishioka T, Amemiya N, Enomoto N, Jiang Z, Yamada Y, Izumi T, Shiohara Y, Saitoh T, Iijima Y, and Kakimoto K 2005 IEEE Trans. Appl. Supercond.15 2843-6

[24] Jiang Z, Amemiya N, Nakahata M, Iijima Y, Kakimoto K, Saitoh T and Shiohara Y 2006 IEEE Trans. Appl. Supercond. 16 85-88

[25] Tsukamoto O 2005 Supercond. Sci. and Technol. 18 596-605

[26] Jiang Z, Amemiya N, Maruyama O and Shiohara Y 2007 Physica C, 463-465 790-4

[27] Poole C P, Farach H A, and Creswick R J 1995 Superconductivity (Academic Press, Inc.) 365 - 400

[28] R.P. Feynmann, R.B. Leighton and M. Sands 1964 Lectures on Physics (Addison-Wesley) 2 Ch.17

[29] Faraday M 1832 Phil. Trans. R. Soc. Lond. 122, 125-62 